\title{
Pricing and Analysis of European Chooser Option Under The Vasicek Interest Rate Model
}

\author{
Yanan Yun*, Lingyun Gao \\ Department of Mathematics, Jinan University, Guangzhou, China \\ Email address: \\ yanan_yun@163.com (Yanan Yun), tgaoly@jnu.edu.cn (Lingyun Gao) \\ ${ }^{*}$ Corresponding author
}

\section{To cite this article:}

Yanan Yun, Lingyun Gao. Pricing and Analysis of European Chooser Option Under The Vasicek Interest Rate Model. International Journal of Theoretical and Applied Mathematics. Vol. 6, No. 2, 2020, pp. 19-27. doi: 10.11648/j.ijtam.20200602.11

Received: April 5, 2020; Accepted: April 22, 2020; Published: May 15, 2020

\begin{abstract}
Based on the modification of some assumptions in the traditional Black-Scholes option pricing model, we construct a model that is closer to the real financial market in this paper. That is to say, in order to make up for the shortages of using the standard Brownian motion to describe the underlying asset price, we use fractional Brownian motion to replace the standard Brownian motion in the traditional Black-Scholes model. At the same time, we assume that the interest rate satisfies the Vasicek interest rate model under fractional Brownian motion. Under the above market model, we use the stochastic analysis method under fractional Brownian motion to obtain the pricing formulae of European simple option and complex option, which generalize the existing conclusions. It is not only can be closer to the actual financial market but also make the research more practical. In addition, since the sensitivity analysis of options refers to the sensitivity or response of options to the change of its determinants, we use numerical methods to analyze the impact of the stock initial price, the chooser date and Hurst parameter on the price of European complex chooser option, which not only verifies the rationality of the pricing formula but also has guiding value for option trading.
\end{abstract}

Keywords: European Chooser Option, Vasicek Model, Fractional Brownian Motion

\section{Introduction}

The chooser options are a kind of strange options that the option holders choose the option as call option or put option at a certain time (i.e. chooser date) during the validity of the option. According to whether strike price and maturity date of the underlying options are the same, the chooser options can be divided into simple chooser option and complex chooser option. In addition, the chooser options can be divided into American option and European option according to whether the underlying options can be exercised in advance. At present, many scholars have been devoted to the study of the pricing of the chooser option. In 1991, reference [1] gave the pricing formula of European standard chooser option under the Black-Sholes model. In 2009, reference [2] discussed the pricing problem of American chooser option. Reference [3] used insurance actuarial method to derive the pricing formula of post-determined option. Reference [4] considered the chooser option pricing of the underlying stock price that satisfies the Heston stochastic volatility model. Reference [5] studied the complex chooser option pricing problem when the stock price follows a continuous generalized exponential O-U process model.

In the above model, the interest rate is a constant or a definite function of time. However, in the real financial market, the interest rate presents randomness, such as the equilibrium interest rate model and the no arbitrage interest rate model [6]. Reference [7] used the partial differential equations models to study the pricing of European gap options under the stochastic interest rate model. Reference [8] studied the pricing of vulnerable options under the jump-diffusion model with the random interest rate.

In recent years, a large number of empirical financial studies have shown that the stock price process has characteristics such as long-term dependence, autocorrelation and "spikes and thick tails". The fractional Brownian motion not only has the above characteristics, but also makes up for the lack of using the standard Brownian motion to describe the underlying asset price. In addition, when $H=\frac{1}{2}$, the fractional Brownian motion is the standard Brownian motion. In reference [9], the pricing formula of European complex 
chooser option is given by using quasi-martingale pricing method with the fractional Brownian motion. Reference [10] studied the pricing of compound options with the random interest rate under the fractional Brownian motion.

In this paper, we consider the stock price obeys the stochastic differential equation under the fractional Brownian motion, and the interest rate obeys the Vasicek interest rate model. By using the stochastic analysis theory, we get the pricing formulae of European simple chooser option and complex chooser option, and generalize the existing pricing model, which can be closer to the actual financial market and make the research more practical.

\section{Preparatory Knowledge}

Definition 1 [11] Let $\left\{\Omega, F,\left(F_{t}\right)_{t \geq 0}, P\right\}$ be a probability space and $H \in(0,1)$ be Hurst parameter. If $\left\{B_{H}(t): t \geq 0\right\}$ satisfies the following conditions, then the continuous Gaussian process $\left\{B_{H}(t): t \geq 0\right\}$ is called the fractional Brownian motion with Hurst parameter:

1) If $B_{H}(t)=0$, then $\mathrm{E}\left[B_{H}(t)\right]=0,(t \geq 0)$,

2) $\mathrm{E}\left[B_{H}(t) B_{H}(s)\right]=\frac{1}{2}\left\{|t|^{2 H}+|s|^{2 H}-|t-s|^{2 H}\right\}$,

where $\mathrm{E}[\cdot]$ is the expectation about probability measure $P$.

Lemma 1 [12] If $(X, Y) \sim N(\mu, \Sigma)$, where

$$
\begin{aligned}
& \mu=\left(\begin{array}{c}
\mu_{X} \\
\mu_{Y}
\end{array}\right), \Sigma=\left(\begin{array}{cc}
\sigma_{X}^{2} & \rho \sigma_{X} \sigma_{Y} \\
\rho \sigma_{X} \sigma_{Y} & \sigma_{Y}^{2}
\end{array}\right) \text {, then } \\
& \text { 1) } \mathrm{E}\left[e^{X} \mathrm{I}_{\{Y \geq b\}}\right]=e^{\mu_{X}+\frac{1}{2} \sigma_{X}^{2}} N\left(\frac{-b+\mu_{Y}+\operatorname{cov}(X, Y)}{\sigma_{Y}}\right), \\
& \mathrm{E}\left[e^{X} N(\varphi(Y)) \mathrm{I}_{\left\{Y b_{3}\right]}\right. \\
& \text { 2) }=e^{\mu_{X}+\frac{1}{2} \sigma_{X}^{2}} \frac{-b+\mu_{Y}+\operatorname{cov}(X, Y)}{\sigma_{Y}} N\left(\varphi\left(-\sigma_{Y} y^{\prime}+\mu_{Y}+\operatorname{cov}(X, Y)\right)\right) \frac{1}{\sqrt{2 \pi}} e^{-\frac{y^{2}}{2}} d y^{\prime},
\end{aligned}
$$

where $N(\bullet)$ is the distribution function of the standard normal distribution, and $\varphi(Y)$ is the function of the random variable $Y$.

\section{Market Model}

Assuming that there are only two kinds of assets in a continuously tradable, frictionless and arbitrage-free financial market, one is risk-free assets (such as bonds), the other is risk assets (such as stocks). The stock price and interest rate meet the following stochastic differential equations respectively

$$
\begin{aligned}
& d S(t)=S(t)\left[(r(t)-q) d t+\sigma_{1} d B_{1}^{H}(t)\right], \\
& d r(t)=k[\theta-r(t)] d t+\sigma_{2} d B_{2}^{H}(t),
\end{aligned}
$$

where $q>0, \sigma_{1}>0$ are constants, which respectively represent the dividend rate of continuous stock payment and the volatility of stock price; $k, \theta, \sigma_{2}$ are constants, and $k$ is the mean regression speed of interest rate; $\left\{B_{1}^{H}(t), t \geq 0\right\}$ and $\left\{B_{2}^{H}(t), t \geq 0\right\}$ are independent fractional Brownian motions on the risk-neutral measurement $\operatorname{space}\left(\Omega, F,\left(F_{t}\right)_{t \geq 0}, P\right)$.

Lemma 2 [10] The solution of the stochastic differential equation (1) is

$$
S(t)=S(0) \exp \left\{\int_{0}^{t} r(s) d s-q t-\frac{1}{2} \sigma_{1}^{2} t^{2 H}+\sigma_{1} B_{1}^{H}(t)\right\} .
$$

Lemma 3 [13] The solution of the stochastic differential equation (2) is

$$
r(s)=r(t) e^{k t-k s}+k \theta \int_{t}^{s} e^{k u-k s} d u+\sigma_{2} \int_{t}^{s} e^{k u-k s} d B_{2}^{H}(u),(0 \leq t \leq s) .
$$

Lemma 4 [14] The call option price with maturity at time $T_{1}$ and strike price $K_{1}$ is $C_{T_{1}, K_{1}}(t, S(t))$ at time $t$, the put option price with maturity at time $T_{2}$ and strike price $K_{2}$ is $P_{T_{2}, K_{2}}(t, S(t))$ at time $t$, then there is a unique $S^{*}$, such that

$$
C_{T_{1}, K_{1}}\left(T, S^{*}\right)=P_{T_{2}, K_{2}}\left(T, S^{*}\right),\left(T \leq T_{1}, T \leq T_{2}\right) .
$$

Theorem 1 Under the market model (1) (2), the pricing formulae of European call option and put option with maturity at time $T$ and strike price $K$ at time $t(0 \leq t \leq T)$ are

$$
\begin{gathered}
C(t, S(t))=S(t) e^{-q(T-t)} N\left(d_{1}\right)-K e^{-D+\frac{1}{2} \sigma_{Y}^{2}} N\left(d_{2}\right), \\
P(t, S(t))=K e^{-D+\frac{1}{2} \sigma_{Y}^{2}} N\left(-d_{2}\right)-S(t) e^{-q(T-t)} N\left(-d_{1}\right),
\end{gathered}
$$

where

$$
\begin{gathered}
d_{1}=\frac{\ln \frac{S(t)}{K}+D-q(T-t)+\frac{1}{2} \sigma_{1}^{2}\left(T^{2 H}-t^{2 H}\right)}{\sqrt{\sigma_{1}^{2}\left(T^{2 H}-t^{2 H}\right)+\sigma_{Y}^{2}}}, \\
d_{2}=d_{1}-\sqrt{\sigma_{1}^{2}\left(T^{2 H}-t^{2 H}\right)+\sigma_{Y}^{2}}, \\
D=\int_{t}^{T} r(t) e^{k t-k s} d s+k \theta \int_{t}^{T} \int_{t}^{s} e^{k u-k s} d u d s, \\
\sigma_{Y}^{2}=2 H \sigma_{2}^{2} \int_{t}^{T}\left(\int_{u}^{T} e^{k u-k s} d s\right)^{2} u^{2 H-1} d u .
\end{gathered}
$$

Proof: According to the theory of risk neutral pricing, the price of European call option with maturity at time $T$ and strike price $K$ at time $t(0 \leq t \leq T)$ is

$$
\begin{aligned}
& C(t, S(t)) \\
& =\mathrm{E}\left[e^{-\int_{t}^{T} r(s) d s}(S(T)-K)^{+}\right] \\
& =\mathrm{E}\left[e^{-\int_{t}^{T} r(s) d s} S(T) \mathbf{I}_{\{S(T) \geq K\}}\right]-\mathrm{E}\left[e^{-\int_{t}^{T} r(s) d s} K \mathrm{I}_{\{S(T) \geq K\}}\right] \\
& \triangleq C_{1}-C_{2} .
\end{aligned}
$$

From Lemma 2 and Lemma 3, we get that

$$
\begin{array}{r}
S(T)=S(t) \exp \left\{\int_{t}^{T} r(s) d s-q(T-t)-\frac{1}{2} \sigma_{1}^{2}\left(T^{2 H}-t^{2 H}\right)+\sigma_{1}\left(B_{1}^{H}(T)-B_{1}^{H}(t)\right)\right\}, \\
\int_{t}^{T} r(s) d s=\int_{t}^{T} r(t) e^{k t-k s} d s+k \theta \int_{t}^{T} \int_{t}^{s} e^{k u-k s} d u d s+\sigma_{2} \int_{t}^{T} \int_{u}^{T} e^{k u-k s} d s d B_{2}^{H}(u) .
\end{array}
$$

Let

$$
\begin{gathered}
X=\sigma_{1}\left(B_{1}^{H}(T)-B_{1}^{H}(t)\right), \\
Y=\sigma_{2} \int_{t}^{T} \int_{u}^{T} e^{k u-k s} d s d B_{2}^{H}(u), \\
Z=X+Y, \\
\sigma_{Y}^{2}=2 H \sigma_{2}^{2} \int_{t}^{T}\left(\int_{u}^{T} e^{k u-k s} d s\right)^{2} u^{2 H-1} d u,
\end{gathered}
$$




$$
\begin{gathered}
D=\int_{t}^{T} r(t) e^{k t-k s} d s+k \theta \int_{t}^{T} \int_{t}^{s} e^{k u-k s} d u d s, \\
d=\ln \frac{K}{S(t)}-D+q(T-t)+\frac{1}{2} \sigma_{1}^{2}\left(T^{2 H}-t^{2 H}\right) .
\end{gathered}
$$

Since $\left\{B_{1}^{H}(t), t \geq 0\right\}$ and $\left\{B_{2}^{H}(t), t \geq 0\right\}$ are independent of each other, $X$ and $Y$ are independent of each other. We have that

$$
\begin{gathered}
X \sim N\left(0, \sigma_{1}^{2}\left(T^{2 H}-t^{2 H}\right)\right), \\
Y \sim N\left(0, \sigma_{Y}^{2}\right), \\
Z \sim N\left(0, \sigma_{1}^{2}\left(T^{2 H}-t^{2 H}\right)+\sigma_{Y}^{2}\right), \\
\operatorname{cov}(X, Z)=\sigma_{1}^{2}\left(T^{2 H}-t^{2 H}\right), \\
\operatorname{cov}(Y, Z)=\sigma_{Y}^{2} .
\end{gathered}
$$

So

$$
S(T) \geq K \Leftrightarrow Z \geq d
$$

Using Lemma 1 (1), we get that

$$
C_{1}=S(t) e^{-q(T-t)} N\left(d_{1}\right),
$$

where

$$
d_{1}=\frac{\ln \frac{S(t)}{K}+D-q(T-t)+\frac{1}{2} \sigma_{1}^{2}\left(T^{2 H}-t^{2 H}\right)}{\sqrt{\sigma_{1}^{2}\left(T^{2 H}-t^{2 H}\right)+\sigma_{Y}^{2}}} .
$$

In the same way, we can get that

$$
C_{2}=K e^{-D+\frac{1}{2} \sigma_{Y}^{2}} N\left(d_{2}\right),
$$

where

$$
d_{2}=d_{1}-\sqrt{\sigma_{1}^{2}\left(T^{2 H}-t^{2 H}\right)+\sigma_{Y}^{2}}
$$

Therefore, the pricing formula for European call option at time $t(0 \leq t \leq T)$ is

$$
C(t, S(t))=S(t) e^{-q(T-t)} N\left(d_{1}\right)-K e^{-D+\frac{1}{2} \sigma_{Y}^{2}} N\left(d_{2}\right) .
$$

According to the parity relationship between European call option and put option, the pricing formula of European put option at time $t(0 \leq t \leq T)$ can be obtained as follows

$$
\begin{aligned}
P(t, S(t))=K e^{-D+\frac{1}{2} \sigma_{Y}^{2}} N\left(-d_{2}\right)-S(t) e^{-q(T-t)} N\left(-d_{1}\right) . & \text { (32) } \quad \text { formula (33), we get that. } \\
c c o(t, S(t)) & =\mathrm{E}\left[e^{-\int_{t}^{T_{c}} r(s) d s} \max \left\{C_{T, K}\left(T_{c}, S\left(T_{c}\right)\right), P_{T, K}\left(T_{c}, S\left(T_{c}\right)\right)\right\}\right] \\
& =\mathrm{E}\left[e^{-\int_{t}^{T_{c}} r(s) d s} \max \left\{C_{T, K}\left(T_{c}, S\left(T_{c}\right)\right), C_{T, K}\left(T_{c}, S\left(T_{c}\right)\right)+K e^{-D_{1}+\frac{1}{2} \sigma_{Y_{1}}^{2}}-S\left(T_{c}\right) e^{-q\left(T-T_{c}\right)}\right\}\right] \\
& =\mathrm{E}\left[e^{-\int_{t}^{T_{c}} r(s) d s} C_{T, K}\left(T_{c}, S\left(T_{c}\right)\right]+\mathrm{E}\left[e^{-\int_{t}^{T_{c}} r(s) d s} \max \left\{0, K e^{-D_{1}+\frac{1}{2} \sigma_{Y_{1}}^{2}}-S\left(T_{c}\right) e^{-q\left(T-T_{c}\right)}\right\}\right]\right. \\
& =\mathrm{E}\left[e^{-\int_{t}^{T} r(s) d s}(S(T)-K)^{+}\right]+\mathrm{E}\left[e^{-\int_{t}^{T_{c}} r(s) d s} \max \left\{0, K e^{-D_{1}+\frac{1}{2} \sigma_{Y_{1}}^{2}}-S\left(T_{c}\right) e^{-q\left(T-T_{c}\right)}\right\}\right],
\end{aligned}
$$

where

$$
\begin{gathered}
d_{1}=\frac{\ln \frac{S(t)}{K}+D-q(T-t)+\frac{1}{2} \sigma_{1}^{2}\left(T^{2 H}-t^{2 H}\right)}{\sqrt{\sigma_{1}^{2}\left(T^{2 H}-t^{2 H}\right)+\sigma_{Y}^{2}}}, \\
d_{2}=d_{1}-\sqrt{\sigma_{1}^{2}\left(T^{2 H}-t^{2 H}\right)+\sigma_{Y}^{2}}, \\
d_{3}=\frac{\ln \frac{S(t)}{K}+D-q(T-t)+\frac{1}{2} \sigma_{1}^{2}\left(T_{c}^{2 H}-t^{2 H}\right)-\frac{1}{2} \sigma_{Y_{1}}^{2}}{\sqrt{\sigma_{1}^{2}\left(T_{c}^{2 H}-t^{2 H}\right)+\sigma_{Y_{2}}^{2}}}, \\
d_{4}=d_{3}-\sqrt{\sigma_{1}^{2}\left(T_{c}^{2 H}-t^{2 H}\right)+\sigma_{Y_{2}}^{2}}, \\
D=\int_{t}^{T} r(t) e^{k t-k s} d s+k \theta \int_{t}^{T} \int_{t}^{s} e^{k u-k s} d u d s, \\
\sigma_{Y}^{2}=2 H \sigma_{2}^{2} \int_{t}^{T}\left(\int_{u}^{T} e^{k u-k s} d s\right)^{2} u^{2 H-1} d u, \\
\sigma_{Y_{1}}^{2}=2 H \sigma_{2}^{2} \int_{t}^{T_{c}}\left(\int_{T_{c}}^{T} e^{k u-k s} d s\right)^{2} u^{2 H-1} d u+2 H \sigma_{2}^{2} \int_{T_{c}}^{T}\left(\int_{u}^{T} e^{k u-k s} d s\right)^{2} u^{2 H-1} d u, \\
\sigma_{Y_{2}}^{2}=2 H \sigma_{2}^{2} \int_{t}^{T_{c}}\left(\int_{u}^{T_{c}} e^{k u-k s} d s\right)^{2} u^{2 H-1} d u .
\end{gathered}
$$

Proof: According to the theory of risk neutral pricing, the parity relationship between European call and put option, and 
where

$$
\begin{gathered}
D_{1}=\int_{T_{c}}^{T} r(t) e^{k t-k s} d s+k \theta \int_{T_{c}}^{T} \int_{t}^{s} e^{k u-k s} d u d s \\
\sigma_{Y_{1}}^{2}=2 H \sigma_{2}^{2} \int_{t}^{T_{c}}\left(\int_{T_{c}}^{T} e^{k u-k s} d s\right)^{2} u^{2 H-1} d u+2 H \sigma_{2}^{2} \int_{T_{c}}^{T}\left(\int_{u}^{T} e^{k u-k s} d s\right)^{2} u^{2 H-1} d u
\end{gathered}
$$

Obviously, the first item in formula (43) is the price of European call option with maturity at time $T$ and strike price $K$, which the calculation result has been given by formula (6). The second item in formula (43) is the price of European put option with the maturity at time $T_{c}$, strike price $K e^{-D_{1}+\frac{1}{2} \sigma_{\gamma_{1}^{2}}}$ and the underlying stock price $S\left(T_{c}\right) e^{-q\left(T-T_{c}\right)}$, which the calculation result has been given by formula (7), i.e.

$$
\begin{aligned}
& \mathrm{E}\left[e^{-\int_{t}^{T_{c}} r(s) d s} \max \left\{0, K e^{-D_{1}+\frac{1}{2} \sigma_{\gamma_{1}}^{2}}-S\left(T_{c}\right) e^{-q\left(T-T_{c}\right)}\right\}\right] \\
& =K e^{-D_{1}+\frac{1}{2} \sigma_{\eta_{1}^{2}}^{2}} e^{-D_{2}+\frac{1}{2} \sigma_{r_{2}}^{2}} N\left(-d_{4}\right)-S(t) e^{-q\left(T-T_{c}\right)} e^{-q\left(T_{c}-t\right)} N\left(-d_{3}\right) \\
& =K e^{-D+\frac{1}{2} \sigma_{r_{1}}^{2}+\frac{1}{2} \sigma_{r_{2}}^{2}} N\left(-d_{4}\right)-S(t) e^{-q(T-t)} N\left(-d_{3}\right),
\end{aligned}
$$

where

$$
d_{3}=\frac{\ln \frac{S(t)}{K}+D-q(T-t)+\frac{1}{2} \sigma_{1}^{2}\left(T_{c}^{2 H}-t^{2 H}\right)-\frac{1}{2} \sigma_{Y_{1}}^{2}}{\sqrt{\sigma_{1}^{2}\left(T_{c}^{2 H}-t^{2 H}\right)+\sigma_{Y_{2}}^{2}}},
$$

$$
d_{4}=d_{3}-\sqrt{\sigma_{1}^{2}\left(T_{c}^{2 H}-t^{2 H}\right)+\sigma_{Y_{2}}^{2}},
$$

$$
\begin{gathered}
D_{2}=\int_{t}^{T_{c}} r(t) e^{k t-k s} d s+k \theta \int_{t}^{T_{c}} \int_{t}^{s} e^{k u-k s} d u d s, \\
\sigma_{Y_{2}}^{2}=2 H \sigma_{2}^{2} \int_{t}^{T_{c}}\left(\int_{u}^{T_{c}} e^{k u-k s} d s\right)^{2} u^{2 H-1} d u .
\end{gathered}
$$

Therefore, the pricing formula of European simple chooser option at time $t\left(0 \leq t \leq T_{c}\right)$ is

$$
\begin{aligned}
& \operatorname{cco}(t, S(t)) \\
& =S(t) e^{-q(T-t)} N\left(d_{1}\right)-K e^{-D+\frac{1}{2} \sigma_{Y}^{2}} N\left(d_{2}\right) \\
& +K e^{-D+\frac{1}{2} \sigma_{Y_{1}}^{2}+\frac{1}{2} \sigma_{Y_{2}}^{2}} N\left(-d_{3}\right)-S(t) e^{-q(T-t)} N\left(-d_{4}\right) .
\end{aligned}
$$

\subsection{The Pricing of European Complex Chooser Option}

If $T_{1} \neq T_{2}$ or $K_{1} \neq K_{2}$, it is called European complex chooser option.

Theorem 3 Under the market model (1) (2), the pricing formula of European complex option at time $t\left(0 \leq t \leq T_{c}\right)$ is:

$$
\begin{aligned}
& C C O(t, S(t)) \\
& =S(t) e^{-q\left(T_{1}-t\right)} N\left(a_{1}, b_{1} ; \rho_{1}\right)-K_{1} e^{-B_{1}+\frac{1}{2} \sigma_{Y}^{2}+\frac{1}{2} \sigma_{\gamma_{1}}^{2}} N\left(a_{2}, b_{2} ; \rho_{1}\right)+K_{2} e^{-B_{2}+\frac{1}{2} \sigma_{Y}^{2}+\frac{1}{2} \sigma_{Y_{2}}^{2}} N\left(-a_{2},-b_{4} ; \rho_{2}\right)-S(t) e^{-q\left(T_{2}-t\right)} N\left(-a_{1},-b_{3} ; \rho_{2}\right),
\end{aligned}
$$

where

$$
\begin{gathered}
a_{1}=\frac{\ln \frac{S(t)}{S^{*}}+B-q\left(T_{c}-t\right)+\frac{1}{2} \sigma_{1}^{2}\left(T_{c}^{2 H}-t^{2 H}\right)}{\sqrt{\sigma_{1}^{2}\left(T_{c}^{2 H}-t^{2 H}\right)+\sigma_{Y}^{2}}}, \\
a_{2}=a_{1}-\sqrt{\sigma_{1}^{2}\left(T_{c}^{2 H}-t^{2 H}\right)+\sigma_{Y}^{2}}, \\
b_{1}=\frac{\ln \frac{S(t)}{K_{1}}+B_{1}-q\left(T_{1}-t\right)+\frac{1}{2} \sigma_{1}^{2}\left(T_{1}^{2 H}-t^{2 H}\right)}{\sqrt{\sigma_{1}^{2}\left(T_{1}^{2 H}-t^{2 H}\right)+\sigma_{Y}^{2}+\sigma_{Y_{1}}^{2}}}, \\
b_{3}=\frac{\ln \frac{S(t)}{K_{2}}+B_{2}-q\left(T_{2}-t\right)+\frac{1}{2} \sigma_{1}^{2}\left(T_{2}^{2 H}-t^{2 H}\right)}{\sqrt{\sigma_{1}^{2}\left(T_{2}^{2 H}-t^{2 H}\right)+\sigma_{Y}^{2}+\sigma_{Y_{2}}^{2}}}, \\
b_{2}=b_{1}-\sqrt{\sigma_{1}^{2}\left(T_{1}^{2 H}-t^{2 H}\right)+\sigma_{Y}^{2}+\sigma_{Y_{1}}^{2}}, \\
b_{4}=\frac{b_{3}-\sqrt{\sigma_{1}^{2}\left(T_{2}^{2 H}-t^{2 H}\right)+\sigma_{Y}^{2}+\sigma_{Y_{2}}^{2}},}{\sqrt{\sigma_{1}^{2}\left(T_{1}^{2 H}-t^{2 H}\right)+\sigma_{Y}^{2}+\sigma_{Y_{1}}^{2}}}, \\
\rho_{2}=\frac{\sqrt{\sigma_{1}^{2}\left(T_{c}^{2 H}-t^{2 H}\right)+\sigma_{Y}^{2}}}{\sqrt{\sigma_{1}^{2}\left(T_{2}^{2 H}-t^{2 H}\right)+\sigma_{Y}^{2}+\sigma_{Y_{2}}^{2}}}, \\
B=\int_{t}^{T_{c}} r(t) e^{k t-k s} d s+k \theta \int_{t}^{T_{c}} \int_{t}^{s} e^{k u-k s} d u d s, \\
B_{1}=\int_{t}^{T_{1}} r(t) e^{k t-k s} d s+k \theta \int_{t}^{T_{1}} \int_{t}^{s} e^{k u-k s} d u d s,
\end{gathered}
$$




$$
\begin{gathered}
B_{2}=\int_{t}^{T_{2}} r(t) e^{k t-k s} d s+k \theta \int_{t}^{T_{2}} \int_{t}^{s} e^{k u-k s} d u d s, \\
\sigma_{Y}^{2}=2 H \sigma_{2}^{2} \int_{t}^{T_{c}}\left(\int_{u}^{T_{c}} e^{k u-k s} d s\right)^{2} u^{2 H-1} d u, \\
\sigma_{Y_{1}}^{2}=2 H \sigma_{2}^{2} \int_{t}^{T_{c}}\left(\int_{T_{c}}^{T_{1}} e^{k u-k s} d s\right)^{2} u^{2 H-1} d u+2 H \sigma_{2}^{2} \int_{T_{c}}^{T_{1}}\left(\int_{u}^{T_{1}} e^{k u-k s s} d s\right)^{2} u^{2 H-1} d u, \\
\sigma_{Y_{2}}^{2}=2 H \sigma_{2}^{2} \int_{t}^{T_{c}}\left(\int_{T_{c}}^{T_{T}} e^{k u-k s} d s\right)^{2} u^{2 H-1} d u+2 H \sigma_{2}^{2} \int_{T_{c}}^{T_{2}}\left(\int_{u}^{T_{2}} e^{k u-k s} d s\right)^{2} u^{2 H-1} d u,
\end{gathered}
$$

$N(x, y ; \rho)$ is the cumulative distribution function of the two-dimensional standard normal distribution.

Proof: According to the theory of risk neutral pricing, formula (33), Lemma 4 and Theorem 1, we have that

$$
\begin{aligned}
& C C O(t, S(t)) \\
& =\mathrm{E}\left[e^{-\int_{t}^{T_{c}} r(s) d s} \max \left\{C_{T_{1}, K_{1}}\left(T_{c}, S\left(T_{c}\right)\right), P_{T_{2}, K_{2}}\left(T_{c}, S\left(T_{c}\right)\right)\right\}\right] \\
& =\mathrm{E}\left[e^{-\int_{t}^{T_{c}} r(s) d s}\left(C_{T_{1}, K_{1}}\left(T_{c}, S\left(T_{c}\right)\right) \mathbf{I}_{\left\{S\left(T_{c}\right) \leq s^{*}\right\}}+P_{T_{2}, K_{2}}\left(T_{c}, S\left(T_{c}\right)\right) \mathbf{I}_{\left\{S\left(T_{c}\right) S^{*}\right\}}\right\}\right] \\
& =\mathrm{E}\left[e^{-\int_{t}^{T_{c}} r(s) d s} S\left(T_{c}\right) e^{-q\left(T_{1}-T_{c}\right)} N\left(d_{1}\left(T_{c}, T_{1}, K_{1}\right)\right) \mathrm{I}_{\left\{S\left(T_{c}\right) \geq S^{*}\right\}}\right]-\mathrm{E}\left[e^{-\int_{c}^{T_{c} r} r(s) d s} K_{1} e^{-D_{1}+\frac{1}{2} \sigma_{r_{1}^{2}}^{2}} N\left(d_{2}\left(T_{c}, T_{1}, K_{1}\right)\right) \mathrm{I}_{\left\{S\left(T_{c}\right) \geq S^{*}\right\}}\right]
\end{aligned}
$$

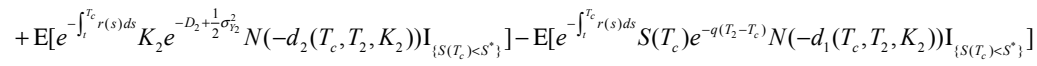

$$
\begin{aligned}
& \triangleq I_{1}-I_{2}+I_{3}-I_{4} \text {, }
\end{aligned}
$$

where

$$
\begin{gathered}
D_{1}=\int_{T_{c}}^{T_{1}} r(t) e^{k t-k s} d s+k \theta \int_{T_{c}}^{T_{1}} \int_{t}^{s} e^{k u-k s} d u d s, \\
D_{2}=\int_{T_{c}}^{T_{2}} r(t) e^{k t-k s} d s+k \theta \int_{T_{c}}^{T_{2}} \int_{t}^{s} e^{k u-k s} d u d s, \\
\sigma_{Y_{1}}^{2}=2 H \sigma_{2}^{2} \int_{t}^{T_{c}}\left(\int_{T_{c}}^{T_{1}} e^{k u-k s} d s\right)^{2} u^{2 H-1} d s+2 H \sigma_{2}^{2} \int_{T_{c}}^{T_{1}}\left(\int_{u}^{T_{1}} e^{k u-k s} d s\right)^{2} u^{2 H-1} d s, \\
\sigma_{Y_{2}}^{2}=2 H \sigma_{2}^{2} \int_{t}^{T_{c}}\left(\int_{T_{c}}^{T_{2}} e^{k u-k s} d s\right)^{2} u^{2 H-1} d s+2 H \sigma_{2}^{2} \int_{T_{c}}^{T_{2}}\left(\int_{u}^{T_{2}} e^{k u-k s} d s\right)^{2} u^{2 H-1} d s .
\end{gathered}
$$

Let

$$
\begin{gathered}
B=\int_{t}^{T_{c}} r(t) e^{k t-k s} d s+k \theta \int_{t}^{T_{c}} \int_{t}^{s} e^{k u-k s} d u d s, \\
d=\ln \frac{S^{*}}{S(t)}-B+q\left(T_{c}-t\right)+\frac{1}{2} \sigma_{1}^{2}\left(T_{c}^{2 H}-t^{2 H}\right), \\
X=\sigma_{1}\left[B_{1}^{H}\left(T_{c}\right)-B_{1}^{H}(t)\right], \\
Y=\sigma_{2} \int_{t}^{T_{c}} \int_{u}^{T_{c}} e^{k u-k s} d s d B_{2}^{H}(u), \\
Z=X+Y, \\
\sigma_{Y}^{2}=2 H \sigma_{2}^{2} \int_{t}^{T_{c}}\left(\int_{u}^{T_{c}} e^{k u-k s} d s\right)^{2} u^{2 H-1} d u .
\end{gathered}
$$

Since $X$ and $Y$ are independent of each other, there are

$$
\begin{gathered}
X \sim N\left(0, \sigma_{1}^{2}\left(T_{c}^{2 H}-t^{2 H}\right)\right), \\
Y \sim N\left(0, \sigma_{Y}^{2}\right), \\
Z \sim N\left(0, \sigma_{1}^{2}\left(T_{c}^{2 H}-t^{2 H}\right)+\sigma_{Y}^{2}\right), \\
\operatorname{cov}(X, Z)=\sigma_{1}^{2}\left(T_{c}^{2 H}-t^{2 H}\right), \\
\operatorname{cov}(Y, Z)=\sigma_{Y}^{2} .
\end{gathered}
$$

So

$$
S\left(T_{c}\right) \geq S^{*} \Leftrightarrow Z \geq d,
$$




$$
S\left(T_{c}\right)<S^{*} \Leftrightarrow Z<d
$$

1) Firstly, we compute $I_{1}, I_{4}$.

$$
\begin{aligned}
I_{1} & =\mathrm{E}\left[e^{-\int_{t}^{T_{c}} r(s) d s} S\left(T_{c}\right) e^{-q\left(T_{1}-t\right)} N\left(d_{1}\left(T_{c}, T_{1}, K_{1}\right)\right) \mathrm{I}_{\left\{S\left(T_{c}\right) \geq S^{*}\right\}}\right] \\
& =S(t) e^{-q\left(T_{1}-t\right)-\frac{1}{2} \sigma^{2}\left(T_{c}^{2 H}-t^{2 H}\right)} \mathrm{E}\left[e^{X} N\left(d_{1}\left(T_{c}, T_{1}, K_{1}\right)\right) \mathrm{I}_{\{Z \geq d\}}\right] .
\end{aligned}
$$

Using lemma 1 (2) to transform $d_{1}\left(T_{c}, T_{1}, K_{1}\right)$ equivalently as follows.

$$
\begin{aligned}
& d_{1}\left(T_{c}, T_{1}, K_{1}\right) \\
& =\frac{\ln \frac{S(t)}{K_{1}}+B_{1}-q\left(T_{1}-t\right)+\frac{1}{2} \sigma_{1}^{2}\left(T_{1}^{2 H}-2 T_{c}^{2 H}+t^{2 H}\right)+Z}{\sqrt{\sigma_{1}^{2}\left(T_{1}^{2 H}-T_{c}^{2 H}\right)+\sigma_{Y_{1}}^{2}}} \\
& =\frac{\ln \frac{S(t)}{K_{1}}+B_{1}-q\left(T_{1}-t\right)+\frac{1}{2} \sigma_{1}^{2}\left(T_{1}^{2 H}-2 T_{c}^{2 H}+t^{2 H}\right)-\sqrt{\sigma_{1}^{2}\left(T_{c}^{2 H}-t^{2 H}\right)+\sigma_{Y}^{2}} Z^{\prime}+\operatorname{cov}(X, Z)}{\sqrt{\sigma_{1}^{2}\left(T_{1}^{2 H}-T_{c}^{2 H}\right)+\sigma_{Y_{1}}^{2}}} \\
& =\frac{\frac{\ln \frac{S(t)}{K_{1}}+B_{1}-q\left(T_{1}-t\right)+\frac{1}{2} \sigma_{1}^{2}\left(T_{1}^{2 H}-t^{2 H}\right)}{\sqrt{\sigma_{1}^{2}\left(T_{1}^{2 H}-t^{2 H}\right)+\sigma_{Y}^{2}+\sigma_{Y_{1}}^{2}}}-\frac{\sqrt{\sigma_{1}^{2}\left(T_{c}^{2 H}-t^{2 H}\right)+\sigma_{Y}^{2}}}{\sqrt{\sigma_{1}^{2}\left(T_{1}^{2 H}-t^{2 H}\right)+\sigma_{Y}^{2}+\sigma_{Y_{1}}^{2}}} Z^{\prime}}{\sqrt{1-\frac{\sigma_{1}^{2}\left(T_{c}^{2 H}-t^{2 H}\right)+\sigma_{Y}^{2}}{\sigma_{1}^{2}\left(T_{1}^{2 H}-t^{2 H}\right)+\sigma_{Y}^{2}+\sigma_{Y_{1}}^{2}}}} \\
& =\frac{b_{1}-\rho_{1} Z^{\prime}}{\sqrt{1-\rho_{1}^{2}}},
\end{aligned}
$$

where

$$
\begin{gathered}
b_{1}=\frac{\ln \frac{S(t)}{K_{1}}+B_{1}-q\left(T_{1}-t\right)+\frac{1}{2} \sigma_{1}^{2}\left(T_{1}^{2 H}-t^{2 H}\right)}{\sqrt{\sigma_{1}^{2}\left(T_{1}^{2 H}-t^{2 H}\right)+\sigma_{Y}^{2}+\sigma_{Y_{1}}^{2}}}, \\
\rho_{1}=\frac{\sqrt{\sigma_{1}^{2}\left(T_{c}^{2 H}-t^{2 H}\right)+\sigma_{Y}^{2}}}{\sqrt{\sigma_{1}^{2}\left(T_{1}^{2 H}-t^{2 H}\right)+\sigma_{Y}^{2}+\sigma_{Y_{1}}^{2}}}, \\
B_{1}=\int_{t}^{T_{1}} r(t) e^{k t-k s} d s+k \theta \int_{t}^{T_{1}} \int_{t}^{s} e^{k u-k s} d u d s .
\end{gathered}
$$

We have that

$$
\begin{aligned}
I_{1} & =S(t) e^{-q\left(T_{1}-t\right)} \int_{-\infty}^{\frac{-d+\operatorname{cov}(X, Z)}{\sqrt{\sigma_{1}^{2}\left(T_{c}^{2 H}-t^{2 H}\right)+\sigma_{Y}^{2}}}} \frac{1}{\sqrt{2 \pi}} e^{-\frac{z^{\prime 2}}{2}} N\left(\frac{b_{1}-\rho_{1} Z^{\prime}}{\sqrt{1-\rho_{1}^{2}}}\right) d z^{\prime} \\
& =S(t) e^{-q\left(T_{1}-t\right)} N\left(a_{1}, b_{1} ; \rho_{1}\right)
\end{aligned}
$$

where

$$
a_{1}=\frac{\ln \frac{S(t)}{S^{*}}+B-q\left(T_{c}-t\right)+\frac{1}{2} \sigma_{1}^{2}\left(T_{c}^{2 H}-t^{2 H}\right)}{\sqrt{\sigma_{1}^{2}\left(T_{c}^{2 H}-t^{2 H}\right)+\sigma_{Y}^{2}}} .
$$

Since

$$
I_{4}=\mathrm{E}\left[e^{-\int_{t}^{T_{c}} r(s) d s} S\left(T_{c}\right) e^{-q\left(T_{2}^{2 H}-T_{c}^{2 H}\right)} N\left(-d_{1}\left(T_{c}, T_{2}, K_{2}\right)\right) \mathrm{I}_{\left\{S\left(T_{c}\right)<S^{*}\right\}}\right],
$$

similar to the calculation process of $I_{1}$, we get that

$$
I_{4}=S(t) e^{-q\left(T_{2}-t\right)} N\left(-a_{1},-b_{3} ; \rho_{2}\right)
$$

where

$$
\begin{gathered}
b_{3}=\frac{\ln \frac{S(t)}{K_{2}}+B_{2}-q\left(T_{2}-t\right)+\frac{1}{2} \sigma_{1}^{2}\left(T_{2}^{2 H}-t^{2 H}\right)}{\sqrt{\sigma_{1}^{2}\left(T_{2}^{2 H}-t^{2 H}\right)+\sigma_{Y}^{2}+\sigma_{Y_{2}}^{2}}}, \\
\rho_{2}=\frac{\sqrt{\sigma_{1}^{2}\left(T_{c}^{2 H}-t^{2 H}\right)+\sigma_{Y}^{2}}}{\sqrt{\sigma_{1}^{2}\left(T_{2}^{2 H}-t^{2 H}\right)+\sigma_{Y}^{2}+\sigma_{Y_{2}}^{2}}},
\end{gathered}
$$




$$
B_{2}=\int_{t}^{T_{2}} r(t) e^{k t-k s} d s+k \theta \int_{t}^{T_{2}} \int_{t}^{s} e^{k u-k s} d u d s
$$

2) Now, we compute $I_{2}, I_{3}$.

$$
\begin{aligned}
I_{2} & =\mathrm{E}\left[e^{-\int_{t}^{T_{c}} r(s) d s} K_{1} e^{-D_{1}+\frac{1}{2} \sigma_{r_{1}}^{2}} N\left(d_{2}\left(T_{c}, T_{1}, K_{1}\right)\right) \mathbf{I}_{\left\{S\left(T_{c}\right) \geq s^{*}\right\}}\right] \\
& =K_{1} e^{-B_{1}+\frac{1}{2} \sigma_{\gamma_{1}^{2}}^{2}} \mathrm{E}\left[e^{-Y} N\left(d_{2}\left(T_{c}, T_{1}, K_{1}\right)\right) \mathbf{I}_{\{Z \geq d\}},\right.
\end{aligned}
$$

Using lemma 1 (2) to transform $d_{2}\left(T_{c}, T_{1}, K_{1}\right)$ equivalently as follows.

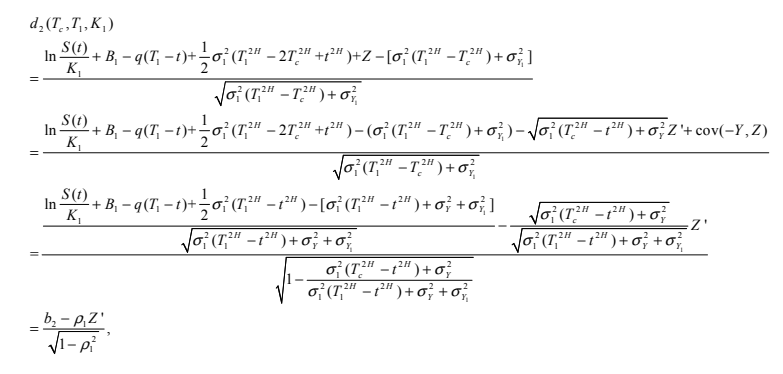

where

$$
b_{2}=b_{1}-\sqrt{\sigma_{1}^{2}\left(T_{1}^{2 H}-t^{2 H}\right)+\sigma_{Y}^{2}+\sigma_{Y_{1}}^{2}} .
$$

We have that

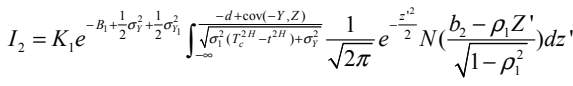

$$
\begin{aligned}
& =K_{1} e^{-B_{1}+\frac{1}{2} \sigma_{Y}^{2}+\frac{1}{2} \sigma_{V_{1}^{2}}^{2}} N\left(a_{2}, b_{2} ; \rho_{1}\right) \text {, }
\end{aligned}
$$

where

$$
a_{2}=a_{1}-\sqrt{\sigma_{1}^{2}\left(T_{c}^{2 H}-t^{2 H}\right)+\sigma_{Y}^{2}}
$$

Since

$$
I_{3}=\mathrm{E}\left[e^{-\int^{T_{c}} r(s) d s} K_{2} e^{-D_{2}+\frac{1}{2} \sigma_{i_{2}}^{2}} N\left(-d_{2}\left(T_{c}, T_{2}, K_{2}\right)\right) \mathrm{I}_{\left\{S\left(T_{c}\right)<S^{*}\right\}}\right],
$$

similar to the calculation process of $I_{2}$, we get that

$$
I_{3}=K_{2} e^{-B_{2}+\frac{1}{2} \sigma_{Y}^{2}+\frac{1}{2} \sigma_{Y_{2}}^{2}} N\left(-a_{2},-b_{4} ; \rho_{2}\right)
$$

where

$$
b_{4}=b_{3}-\sqrt{\sigma_{1}^{2}\left(T_{2}^{2 H}-t^{2 H}\right)+\sigma_{Y}^{2}+\sigma_{Y_{2}}^{2}} .
$$

Therefore, the pricing formula for European complex chooser option at time $t\left(0 \leq t \leq T_{c}\right)$ is

$$
\begin{aligned}
& C C O(t, S(t)) \\
& =S(t) e^{-q\left(T_{1}-t\right)} N\left(a_{1}, b_{1} ; \rho_{1}\right)-K_{1} e^{-B_{1}+\frac{1}{2} \sigma_{Y}^{2}+\frac{1}{2} \sigma_{\sigma_{1}^{2}}^{2}} N\left(a_{2}, b_{2} ; \rho_{1}\right) \\
& +K_{2} e^{-B_{2}+\frac{1}{2} \sigma_{Y}^{2}+\frac{1}{2} \sigma_{\Sigma_{2}^{2}}^{2}} N\left(-a_{2},-b_{4} ; \rho_{2}\right)-S(t) e^{-q\left(T_{2}-t\right)} N\left(-a_{1},-b_{3} ; \rho_{2}\right) .
\end{aligned}
$$

\subsection{Inference}

Inference 1 When $k \rightarrow 0, \theta=0, \sigma_{2}=0$, Theorem 2 is the result of Theorem 5 in reference [15]. Especially, when $H=\frac{1}{2}$ Theorem 2 is the result of reference [16].

Inference 2 When $k \rightarrow 0, \theta=0, \sigma_{2}=0, q=0$, Theorem 3 is the result of Theorem 1 in reference [9]. In particular, when ${ }_{H=\frac{1}{2}}$, Theorem 3 is the pricing formula of European complex options under the standard Brownian motion.

\section{Numerical Analysis}

In this section, we use numerical methods to explain the influence of the initial stock price $S_{0}$, the chooser date $T_{c}$ and Hurst parameter $H$ on European complex chooser option price. The parameter values in model (1) (2) are shown in Table 1.

Table 1. Parameter values in model

\begin{tabular}{lllllllllll}
\hline Parameter & $\boldsymbol{K}_{\mathbf{1}}$ & $\boldsymbol{K}_{\mathbf{2}}$ & $\boldsymbol{T}_{\mathbf{1}}$ & $\boldsymbol{T}_{\mathbf{1}}$ & $\boldsymbol{t}$ & $\boldsymbol{r}_{\mathbf{0}}$ & $\boldsymbol{q}$ & $\boldsymbol{\sigma}_{\mathbf{1}}$ & $\boldsymbol{k}$ & $\boldsymbol{\theta}$ \\
\hline Value & 100 & 90 & 4 & 5 & 0 & 0.05 & 0.02 & 0.4 & 2.4 & 0.03 \\
\hline
\end{tabular}

Table 2 compares the option prices under the fractional Black Scholes model (see [15]) and the Vasicek interest rate model when the chooser date of complex chooser option is $T_{c}=2$. Compared with the pricing formula under the fractional Black-Scholes model, the pricing formula derived by Theorem 3 in this paper that considers the mean reversion of interest rate, which makes the pricing result more consistent with the real financial environment. It can be found that the option price obtained by Theorem 3 has great differences with the pricing result of reference [15], that is to say, the result of the Vasicek interest rate model is higher than the fractional Black Scholes model. In addition, when Hurst parameter is determined, as the stock price ${ }_{S_{0}}$ increases, the price of chooser option decreases first and then increases, which is beneficial for option holders to buy or sell stock at an appropriate time. Finally, when the stock price $s_{0}$ is determined, as Hurst parameter increases, the price of chooser option increases. 
Table 2. Comparison of the influence of stock price $S_{0}$ on option price CCO

\begin{tabular}{lllllll}
\hline \multirow{2}{*}{ Stock Price } & \multicolumn{5}{l}{ Fractional Black-Scholes } & \multicolumn{3}{l}{ Vasicek } \\
\cline { 2 - 7 } & $\boldsymbol{H}_{\mathbf{1}}=\mathbf{0 . 3}$ & $\boldsymbol{H}_{\mathbf{1}}=\mathbf{0 . 5}$ & $\boldsymbol{H}_{\mathbf{1}}=\mathbf{0 . 7}$ & $\boldsymbol{H}_{\mathbf{1}}=\mathbf{0 . 3}$ & $\boldsymbol{H}_{\mathbf{1}}=\mathbf{0 . 5}$ & $\boldsymbol{H}_{\mathbf{1}}=\mathbf{0 . 7}$ \\
\hline 30 & 43.9429 & 45.9352 & 48.8785 & 58.8408 & 60.3617 & 63.9781 \\
40 & 37.0911 & 40.6834 & 44.8456 & 51.3769 & 54.8254 & 60.3052 \\
50 & 32.0754 & 37.2571 & 42.5435 & 45.5959 & 51.1685 & 57.7804 \\
60 & 29.0892 & 35.6296 & 41.9106 & 41.6870 & 49.1123 & 56.2059 \\
70 & 28.0898 & 35.6595 & 42.7778 & 39.5570 & 48.3925 & 55.5405 \\
80 & 28.8933 & 37.1502 & 44.9390 & 39.0622 & 48.8148 & 55.7819 \\
90 & 31.2555 & 39.8931 & 48.1909 & 39.9981 & 50.2416 & 56.9181 \\
100 & 34.9237 & 43.6906 & 52.3511 & 42.1768 & 52.5684 & 58.9151 \\
110 & 39.6646 & 48.3671 & 57.2636 & 45.4282 & 55.7076 & 61.7205 \\
120 & 45.2750 & 53.7722 & 62.7983 & 49.6012 & 59.5799 & 65.2698 \\
\hline
\end{tabular}

Taking stock price $s_{0}=80$ for example. Figure 1 analyzes the influence of chooser date $T_{c}$ on the price of complex chooser option. It can be found that when $T_{c}$ changes from 1.1 to 2 , as Hurst parameter $H$ increases, the price of complex chooser option increases.

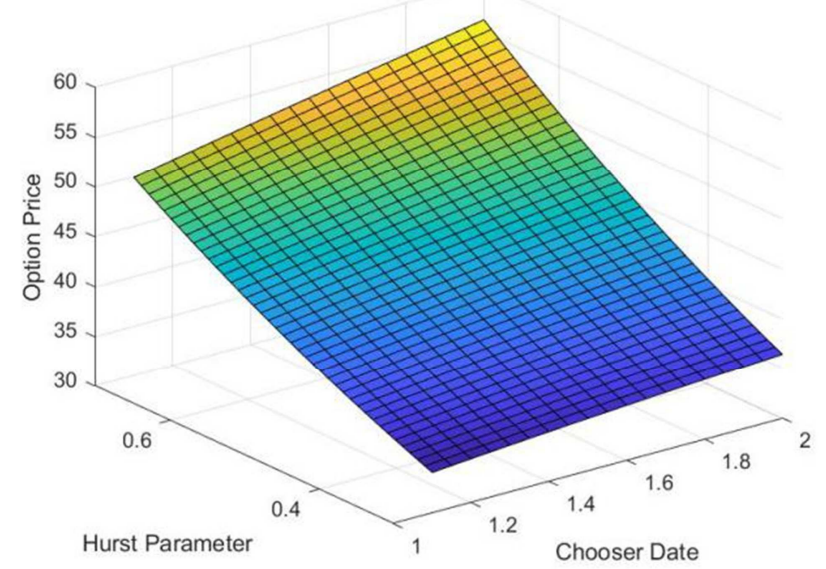

Figure 1. The influence of chooser date $T_{c}$ and Hurst parameter ${ }_{H}$ on option price cco under the Vasicek Model.

\section{Conclusion}

In this paper, we consider two cases: interest rate is stochastic and asset price is stochastic differential equation driven by fractional Brownian motion. The rationality of Theorem 3 is verified by numerical analysis. The results show that Theorem 3 can be used to solve the option price of European complex chooser option when the interest rate and stock price are stochastic differential equations under fractional Brownian motion, and generalize the conclusions in some literature, which makes the option price closer to the actual financial market and has guiding value in option trading.

\section{References}

[1] Rubinstein M. Options for the undecided [J]. Risk, 1991, 4 (4): 43.
[2] Detemple J, Emmerling T. American chooser options [J]. Journal of Economic Dynamics Control, 2009, 33 (2): 128-153.

[3] Xuehui Bi, Xueqiao Du. Actuarial pricing of post-determined options [J]. Journal of Hefei University of Technology, 2007, 30 (5): 649-651.

[4] Guohe Deng. European chooser option pricing and hedging strategy based on Heston model [J]. Journal of Guangxi Normal University (Natural Science Edition), 2012, 30 (3): 36-43.

[5] Jiangjiang Dong, Kai Gao, Xueru Liu. Complex chooser option pricing for continuous O-U process [J]. Journal of Nanjing Normal University (Natural Science Edition), 2018, 42 (2): 16-22.

[6] John C Hull. Options, futures and other derivatives [M]. Beijing: Machinery Industry Press, 2011.

[7] Yan Zhang, Shengwu Zhou, Miao Han, Xinli Suo. European gap option pricing under Vasicek stochastic interest rate model [J]. College Mathematics, 2012, 28 (4): 98-101.

[8] Sang Wu, Chao Xu, Yinghui Dong. Pricing of vulnerable options under jump-diffusion model with random interest rate [J]. Journal of Applied Mathematics, 2019, 42 (4): 518-532.

[9] Yingxin Zhan, Yun Xu. Pricing of European complex chooser option under fractional Brownian motion [J]. Mathematical Theory and Application, 2010, 30 (3): 78-82.

[10] Shucai Yang, Hong Xue, Xiaodong Xue. Fractional compound option pricing model with stochastic interest rate [J]. Journal of Harbin University of Commerce (Natural Sciences Edition), 2014, 30 (1): 98-102.

[11] Ciprian Necula. Option Pricing in a Fractional Brownian Motion Environment [J]. Pure mathematics, 2002, 2 (1): 63-68.

[12] Zhang S, Yuan S, Wang L. Prices of Asian options under stochastic rates [J]. Applied Mathematics- A Journal of Chinese Universities, 2006, 21 (2): 135-142.

[13] Jie Lin, Hong Xue, Xiaodong Wang. Pricing model of gap options under fractional Brownian motion [J]. Journal of Harbin University of Commerce (Natural Sciences Edition), 2012, 28 (5): 616-619.

[14] Korn R, Korn E. Option Pricing and Portfolio Optimization [M]. New York: American Mathematical Society, 2000. 
[15] Shaoqun Liu, Xiangqun Yang. Pricing European contingent claims under fractional Brownian motion [J]. Chinese Journal of applied probability and statistics, 2004, 20 (4): 429-434.
[16] Songnan Chen. Financial engineering [M]. Shanghai: Fudan University Press, 2002. 